\title{
Fluctuation effects in initial conditions for hydrodynamics
}

\author{
Samya Paiva* and Yogiro Hama \\ Instituto de Física, Universidade de São Paulo, Caixa Postal 66318, 05315-970 São Paulo-SP, Brazil \\ Takeshi Kodama \\ Instituto de Física, Universidade do Rio de Janeiro, Caixa Postal 68528, 21945-970 Rio de Janeiro-RJ, Brazil
}

(Received 28 May 1996)

\begin{abstract}
We have studied the fluctuation effects in proton-proton collisions through the analysis of their observables. To investigate the role of fluctuations in the initial conditions, we have used the interacting gluon model, modified by the inclusion of the impact parameter, and have applied the one-dimensional Landau's Hydrodynamical Model to the fireballs thus generated. The rapidity and pseudorapidity distributions were calculated using two distinct procedures, one taking the fluctuations into account and the other the usual method considering only one fireball with the average initial conditions. The results show indeed the importance of fluctuations. [S0556-2813(97)02902-6]
\end{abstract}

PACS number(s): 25.75.-q, 12.40.Ee, 13.85.Hd, 24.10.Nz

\section{INTRODUCTION}

It is well known that the hydrodynamical models usually describe well the various aspects of the multiple particle production phenomena in high-energy nuclear and hadronic collisions. Nevertheless, a simple application of these models may fail when we try to analyze in detail the experimental data that carry information about fluctuations in the system. In a given experimental setup, even under the same initial condition of colliding objects, events with different finalstate configurations take place giving rise to different multiplicities, inelasticities, particle spectra, and so on. This variety - or fluctuation, has either a quantum mechanical or statistical origin or even associated with the impact parameter. Usually, the so-called inclusive data for the final particle distribution is the average over such event-to-event fluctuations for a given experimental initial condition. Let us denote such an averaging process by

$$
\bar{\cdots}=\frac{1}{N} \sum_{j=1}^{N}(\cdots)_{j},
$$

where $N$ is the total number of events and $(\cdots)_{j}$ is the experimental value of some relevant quantity in the $j$ th event. On the other hand, the hydrodynamic models also deal mainly with collective quantities such as density, mean energy, and entropy, which are average values in the momentum space over a statistical ensemble. Let us denote this averaging process by $\langle\cdots\rangle$. In the usual application of hydrodynamic models to describing the inclusive data, we presumably expect, by means of a sort of ergodic assumption $[1]$, that the statistical ensemble average $\langle\ldots\rangle$ substitutes the average over event-to-event fluctuations $\cdots$, that is,

$$
\bar{\cdots} \simeq\langle\cdots\rangle
$$

\footnotetext{
*Present address: Instituto de Física Teórica, UNESP, Rua Pamplona 145, 01405-901 São Paulo - SP, Brazil.
}

However, not all the averages of physical fluctuations can be expressed in terms of the above average over statistical ensemble of the constituent configurations. For example, the quantum-mechanical and impact-parameter fluctuations that occur in the initial condition of each event can never be averaged out with the use of the ergodic hypothesis. The main aim of this report is to discuss the effects of such fluctuations on the observed quantities in a hydrodynamical description.

When we want to introduce fluctuations in the initial conditions of a hydrodynamic system, we must go beyond the hydrodynamic degrees of freedom. They should be calculated from some microscopic model. For this goal, we use here the interacting gluon model [2] (IGM). This is a simple model, which takes into account in the usual way both the gluon distribution in the incident particles and their collision cross section [3], allowing to compute the energy and momentum distribution of the central fireballs on a probabilistic ground. So, it is a made-to-order model for our purpose. In addition to the dynamical fluctuation of the microscopic degrees of freedom, we would also like to include the impactparameter fluctuation. Quantum mechanically, it is in principle impossible to fix the impact parameter. Even if we could theoretically define the trajectories of the incident particles like in heavy-ion collisions where the incident objects are nearly classical, it would equally not be possible in practice to fix the impact parameter due to the actual experimental conditions. We may recall that there exist some experimental techniques to discriminate the central from the peripheral collisions in such reactions. But they do not eliminate fluctuations. So, in any realistic description of nuclear and hadronic collisions, the impact-parameter fluctuation has to be taken into account. Thus, we modify the original IGM to take account of this effect. The fluctuations we are mentioning become especially important in hadronic collisions rather than in heavy-ion collisions. So, in this paper we shall mainly be concerned with $p p$ and $\bar{p} p$ collisions.

In high-energy $p p$ and $\bar{p} p$ collisions, it is probable that the inelasticity, that is, the fraction of the incident energy used to produce the final particles be determined before the 
hydrodynamic scenario sets in. In other words, the inelasticity is the input for the hydrodynamics. We thus calculate the inelasticity distribution using the impact-parameter dependent IGM. To have a first look on the effect of impact parameter, we compare the inelasticity distribution calculated by the IGM with and without the impact-parameter fluctuation. A sizable change in inelasticity distribution, as well as in the leading-particle spectrum leads us to verify the sensitivity of these observables with impact parameter fluctuation. A better agreement of our results with data suggests that we are in the right way.

After introducing the initial conditions given by our IGM, the next step is to choose some hydrodynamic model and study the fluctuation effects on the final particle spectra. For this, we have chosen the one-dimensional Landau's hydrodynamical model [4,5]. In this model, an analytical solution can be obtained over the whole kinematical region just in terms of the invariant fireball mass. This enormously simplifies our task of averaging over all the fluctuations considered.

In order to quantitatively analyze the consequences of these fluctuations, we calculate the rapidity distributions in two distinct ways. The first procedure consists in taking first the average of fireballs over all the fluctuations given by the IGM and then calculate the rapidity distribution applying the hydrodynamics to this unique averaged fireball. This process would correspond to the usual application of a hydrodynamical model. In the second scheme, we apply the hydrodynamics for each event with fluctuating initial conditions to obtain the event-by-event rapidity distributions and then, by summing up these distributions over all the events, calculate the averaged rapidity distribution. The comparison of the above two results reveals us that the rapidity distribution is very sensitive to these fluctuations, pointing out the importance of taking care of them.

In what follows, we present in Sec. II the basic ideas of the IGM, modified by the inclusion of the impact parameter, and the calculation of the initial conditions. The results of the inelasticity distribution and the leading-particle spectrum are also shown. In Sec. III, we show the implementation of the initial conditions in Landau's hydrodynamical model and calculate the rapidity and pseudorapidity distributions. Our conclusions are given in Sec. IV.

\section{FLUCTUATIONS IN THE INITIAL CONDITIONS}

One of the present problems which high-energy nuclear and hadronic collisions are faced with is the determination of the energy deposited in the reaction or, equivalently, the fraction $k$ of the total incident energy $\sqrt{s}$ consumed to produce particles. As mentioned in the Introduction, this fraction, or inelasticity, is an essential ingredient for statistical models of high-energy hadronic collisions. We apply the IGM to calculate this quantity. The main reason for this is that, in terms of few parameters, it allows us to obtain analytically the inelasticity distribution as a function of the incident energy; this, in its turn, is immediately related to the leading-particle spectrum. Another reason would be a very good agreement with the existing data on both these quantities, as will be seen in this section with our formulation of IGM.
The IGM is based on an idea [6] that, in high-energy hadronic collisions, valence quarks weakly interact so that they almost pass thorough, whereas gluons interact strongly. To be more specific [2], the valence quarks are supposed to be responsible for the fragmentation regions, while the interacting gluons produce an indefinite number of mini fireballs through gluon fusion, which eventually form a unique large fireball in the central region. A further simplification in this model can be made by assuming that there is no fragmentation of valence quarks, that is, all the remaining energy not deposited in the central fireball is to be found in the leading particles. We shall adopt this version in this paper. In [2], no reference is made on the impact parameter. Here, we reformulate the model to introduce this new kind of fluctuation for the description of proton-proton and nucleus-nucleus collisions.

\section{A. Impact-parameter fluctuation}

The role of the impact parameter in providing the initial condition for a hydrodynamical model is twofold. First, a given impact parameter $\vec{b}$ defines the probability density of occurrence of a reaction. Then, if a reaction takes place, it determines how do the mass and momentum fluctuate in the initial conditions of the hydrodynamics. To account for the first point, the best way to introduce the impact parameter in quantum-mechanical reaction process is the use of the Eikonal formalism [7]. In the impact-parameter representation, the total inelastic proton-proton cross section can be written as

$$
\int d \vec{b} F(\vec{b})=\sigma_{p p}^{\text {inel }}(\sqrt{s}),
$$

where the incident-energy dependence of the inelastic cross section is well expressed as [8]

$$
\sigma_{p p}^{\mathrm{inel}}=56(\sqrt{s})^{-1.12}+18.16(\sqrt{s})^{0.16}
$$

The function $F(\vec{b})$ is nothing but the partial cross section with respect to the impact parameter $\vec{b}$, which may be written

$$
F(\vec{b})=1-|S(\vec{b})|^{2}
$$

In the IGM, where the inelastic processes are assumed to occur due to the gluon-gluon fusion, we write the Eikonal function as the convolution of the projectile and target gluon thickness functions. Thus, we write

$$
\begin{aligned}
|S(\vec{b})|^{2}= & \exp \left\{-C \int d \vec{b}^{\prime} \int d \vec{b}^{\prime \prime} D\left(\vec{b}^{\prime}\right) D\left(\vec{b}^{\prime \prime}\right)\right. \\
& \left.\times f\left(\vec{b}+\vec{b}^{\prime}-\vec{b}^{\prime \prime}\right)\right\},
\end{aligned}
$$

where $D(\vec{b})$ is the proton thickness function and $C$ is a parameter which should be determined by the normalization condition (3). For $D(\vec{b})$ we take here a Gaussian distribution with the range equal to the proton radius. The function $f(\vec{b})$ in Eq. (5) accounts for the finite interaction range of the gluons and is subject to the constraint 


$$
\int f(\vec{b}) d \vec{b}=1
$$

The simplest choice of $f(\vec{b})$ would be $\delta(\vec{b})$, which represents a point interaction, but it is not consistent (especially in the case of $p p$ collision) with the finite range of the strong interaction. We preferred to parametrize it as a Gaussian with a range $\approx 0.8 \mathrm{fm}$, which gives also a better agreement with the data. So we have eventually

$$
D(\vec{b})=f(\vec{b})=\frac{a}{\pi} e^{-a b^{2}}
$$

with $a=3 / 2 R_{p}^{2}$, where $R_{P} \approx 0.8 \mathrm{fm}$ is the proton radius. We get, then

$$
F(\vec{b})=1-\exp \left\{-\left[\frac{a C}{3 \pi}\right] \exp \left(-\frac{a \vec{b}^{2}}{3}\right)\right\}
$$

\section{B. IGM with impact parameter fluctuation}

Now, having occurred a reaction by gluon exchange at an impact parameter $\vec{b}$, we assume, as in the original IGM [2], that the colliding protons form a central fireball, depositing in it fractions respectively $x(\vec{b})$ and $y(\vec{b})$ of their momenta. Let $n_{i}$ be the number of gluon pairs that carry fractions $x_{i}$ and $y_{i}$. The fractions $x(\vec{b})$ and $y(\vec{b})$ are thus sums over all such gluon pairs

$$
\sum_{i} n_{i} x_{i}=x(\vec{b})
$$

and

$$
\sum_{i} n_{i} y_{i}=y(\vec{b})
$$

From now on, we omit the explicit $\vec{b}$ dependence of $x$ and $y$ in order not to overload the notation, if otherwise necessary. The energy and momentum of the central fireball in the center-of-mass frame of protons are given by

$$
\begin{aligned}
& E(x, y)=\frac{\sqrt{s}}{2}(x+y), \\
& P(x, y)=\frac{\sqrt{s}}{2}(x-y)
\end{aligned}
$$

and its invariant mass $M$ and rapidity $Y$ are

$$
M=\sqrt{s x y} \equiv k \sqrt{s} \quad \text { and } Y=\frac{1}{2} \ln \frac{x}{y} .
$$

As in [2], we can then express the probability of forming a fireball with the specific energy and momentum as a sum over all the sets of gluon pairs $\left\{n_{i}\right\}$ which satisfy the relations (9),

$$
\begin{aligned}
\Gamma(x, y ; \vec{b})= & \sum_{\left\{n_{i}\right\}} \delta\left[x(\vec{b})-\sum_{i} n_{i} x_{i}\right] \\
& \times \delta\left[y(\vec{b})-\sum_{i} n_{i} y_{i}\right] \prod_{i} \mathcal{P}\left(n_{i}\right),
\end{aligned}
$$

where $\mathcal{P}\left(n_{i}\right)$ is the probability of occurring fusions of $n_{i}$ gluon pairs $\left\{x_{i}, y_{i}\right\}$. If these fusions are independent, we may take $\mathcal{P}\left(n_{i}\right)$ as a Poisson distribution

$$
\mathcal{P}\left(n_{i}\right)=\frac{\vec{n}_{i}^{n_{i}} e^{-\bar{n}_{i}}}{n_{i} !}
$$

Note that Eq. (12) is normalized,

$$
\int d x \int d y \Gamma(x, y ; \vec{b})=1 .
$$

Now, expressing the delta functions by Fourier integrals, one can perform all summations in Eq. (12) and arrive at

$$
\begin{aligned}
\Gamma(x, y ; \vec{b})= & \frac{1}{(2 \pi)^{2}} \int_{-\infty}^{+\infty} d u \int_{-\infty}^{+\infty} d s e^{i(u x+s y)} \\
& \times \exp \left\{\sum_{j} \bar{n}_{j}\left[e^{-i\left(u x_{j}+s y_{j}\right)}-1\right]\right\} .
\end{aligned}
$$

At this stage, we shift to the continuous limit from the discrete version considered above, by replacing the sum in Eq. (14) by an integral,

$$
\sum_{j} \overline{n_{j}} \rightarrow \int_{0}^{1} d x^{\prime} \int_{0}^{1} d y^{\prime} w\left(x^{\prime}, y^{\prime} ; \vec{b}\right),
$$

where $w(x, y ; \vec{b})$ is the density of gluon pairs that fuse contributing to the final fireball, with the fraction $x$ of the projectile and $y$ of the target proton momenta, respectively. This quantity is the central ingredient in the present version of the model. In the impact-parameter representation, we may express it as

$$
\begin{aligned}
w(x, y ; \vec{b}) \equiv & \int G\left(x, \vec{b}^{\prime}\right) G\left(y, \vec{b}^{\prime \prime}\right) \frac{\sigma_{g g}(x, y)}{\sigma_{p p}^{\text {inel }}} \\
& \times f\left(\vec{b}+\vec{b}^{\prime}-\vec{b}^{\prime \prime}\right) d \vec{b}^{\prime} d \vec{b}^{\prime \prime}
\end{aligned}
$$

where $G(x, \vec{b})$ and $G(y, \vec{b})$ are the momentum distributions of gluons in the projectile and target protons at a transverse position $\vec{b}$ relative to their symmetry axes, and $\sigma_{g g}(x, y, s)$ is the gluon-gluon interaction cross section. The function $f$ is the same one as in Eq. (5). In this work, we assume that the $x$ and $\vec{b}$ dependences of $G$ factorize, that is, we take

$$
G(x, \vec{b})=\frac{1}{x} D(\vec{b})
$$

and

$$
G(y, \vec{b})=\frac{1}{y} D(\vec{b})
$$


where $D(\vec{b})$ is the proton thickness function appeared before, and the gluon-gluon cross section is parametrized [2] as

$$
\sigma_{g g}(x, y)=\frac{c}{x y s}
$$

where $c$ is a parameter of the model.

Because of the form of $G(x, \vec{b})$ and $\sigma_{g g}$, the spectral function $w(x, y ; \vec{b})$ is sharply peaked at small $(x, y)$ which justifies the approximation

$$
\exp \left[-i\left(u x^{\prime}+s y^{\prime}\right)\right]-1 \simeq-i\left(u x^{\prime}+s y^{\prime}\right)-\frac{1}{2}\left(u x^{\prime}+s y^{\prime}\right)^{2}
$$

in Eq. (14). With this approximation, one obtains an analytical form for $\Gamma(x, y ; \vec{b})$ given by

$$
\Gamma(x, y ; \vec{b}) \simeq \frac{1}{\pi \sqrt{\operatorname{det}(\mathbf{G})}} \exp \left\{-\overrightarrow{\mathbf{X}}^{T} \mathbf{G}^{-1} \overrightarrow{\mathbf{X}}\right\},
$$

where

$$
\mathbf{X}=\left(\begin{array}{c}
x-\langle x\rangle \\
y-\langle y\rangle
\end{array}\right), \mathbf{G}=2\left(\begin{array}{cc}
\left\langle x^{2}\right\rangle & \langle x y\rangle \\
\langle x y\rangle & \left\langle y^{2}\right\rangle
\end{array}\right)
$$

with the notation,

$$
\left\langle x^{m} y^{n}\right\rangle=\int_{0}^{1} d x^{\prime} \int_{0}^{1} d y^{\prime} x^{\prime m} y^{\prime n} w\left(x^{\prime}, y^{\prime} ; \vec{b}\right) .
$$

In terms of the energy and the momentum of the fireball, the probability density reads

$$
\Gamma(E, P ; \vec{b}) \simeq \frac{2}{\sqrt{\pi^{2} / a_{1} a_{2}}} \exp \left\{-a_{1}(E-\langle E\rangle)^{2}-a_{2} P^{2}\right\},
$$

where

$$
a_{1}=\left[s\left(\left\langle x^{2}\right\rangle+\langle x y\rangle\right)\right]^{-1}, \quad a_{2}=\left[s\left(\left\langle x^{2}\right\rangle-\langle x y\rangle\right)\right]^{-1}
$$

and

$$
\langle E\rangle=\frac{\sqrt{s}}{2}(\langle x\rangle+\langle y\rangle)
$$

Note that (apparently) Eq. (23) is still normalized,

$$
\int d E \int d P \Gamma(E, P ; \vec{b})=1 .
$$

The expression (20) or (23) describes the relative probability of formation, at a given impact parameter, of a central fireball with energy $E$ and momentum $P$. In these expressions, however, no constraint has been imposed neither on the fireball mass $M$ nor on its momentum $P$. Actually, there are the natural upper limits on these variables implied by the energy-momentum conservation. There is also a minimum allowed fireball mass $M_{\min }$, which we shall fix to be $2 m_{\pi}$. So, in order to recover the correct normalization, we have to put some additional factor in Eq. (23). Combining this with the probability of occurrence of a reaction with a given impact parameter as discussed in Sec. II A, we finally write the probability $\chi(E, P ; \vec{b})$ of having a fireball with energy $E$ and momentum $P$ at an impact parameter $\vec{b}$ as

$$
\chi(E, P ; \vec{b})=\chi_{0}(\vec{b}) \Gamma(E, P ; \vec{b})
$$

where $\chi_{0}(\vec{b})$ should be determined by the condition

$$
\int d E \int d P \chi(E, P ; \vec{b}) \theta\left(\sqrt{\left(E^{2}-P^{2}\right) / s}-k_{\text {min }}\right)=\frac{1}{\sigma_{p p}^{\text {inel }}} F(\vec{b}),
$$

where $k_{\min }$ is related to the minimum fireball mass $M_{\text {min }}$ through

$$
k_{\min }=\frac{M_{\min }}{\sqrt{s}} .
$$

The expression (27) shows that, when the matter overlap is small (corresponding to a large impact parameter), not only the average fireball mass or the inelasticity is small as implied by Eq. (23), but also the probability of such fireball formation is small. This is a reflection of quantum effects in impact parameter and has shown to be crucial in our description.

\section{Inelasticity distribution}

Now we have all the necessary ingredients for the obtention of $\chi(E, P ; \vec{b})$, our generator function of the initial conditions, which includes the energy, momentum, and impact parameter fluctuations. Note that, in comparison with the original model, we have introduced a new kind of fluctuation without including any additional free parameter, except for the geometrical radius of the proton $R_{p}$ which appears in Eq. (8).

Once $\chi(E, P ; \vec{b})$ has been obtained, the inelasticity distribution $\chi(k)$ can easily be calculated by integrating it over $E, P$, and $\vec{b}$ with the inelasticity $k=\sqrt{\left(E^{2}-P^{2}\right) / s}$ fixed, namely

$$
\begin{aligned}
\chi(k)= & \int d \vec{b} \int d E \int d P \chi(E, P ; \vec{b}) \\
& \times \delta\left(\sqrt{\left(E^{2}-P^{2}\right) / s}-k\right) \theta\left(\sqrt{\left(E^{2}-P^{2}\right) / s}-k_{\min }\right) .
\end{aligned}
$$

As for the leading-particle spectrum, assuming an approximate factorization of $x_{L}\left(=2 p_{L} / \sqrt{s}\right)$ and $p_{T}$ dependences, we have

$$
E_{L} \frac{d^{3} \sigma}{d p^{3}} \approx f\left(x_{L}\right) h\left(p_{T}\right)
$$

where 


$$
\begin{aligned}
f\left(p_{L}\right)= & \int d \vec{b} \int d P \int d E \chi(E, P ; \vec{b}) \\
& \times \theta\left(\sqrt{\left(E^{2}-P^{2}\right) / s}-k_{\min }\right) \delta\left(\frac{\sqrt{s}-(E+P)}{2}-p_{L}\right)
\end{aligned}
$$

and we have parametrized [9]

$$
h\left(p_{T}\right)=\frac{\beta^{2}}{2 \pi} e^{-\beta p_{T}} ; \quad \beta \approx 4.0 \mathrm{GeV}^{-1}
$$

The only experimental information available on $\chi(k)$ at the present moment is the one extracted from [10] corresponding to $\sqrt{s}=16.5 \mathrm{GeV}$. In this reference, what is presented is the unnormalized cross section measured in the range $0.08 \leqslant k \leqslant 0.72$. However, both the result of [2] and ours are normalized curves in the whole $k$ range $\left(k_{\min } \leqslant k \leqslant 1\right)$. In order to correctly compare our result with the data, we gave the latter the same normalization as our curve in the range where they have been measured. We show in Fig. 1 the comparison of our result with the experimental data together with the curve obtained in [2], i.e., without the impact-parameter fluctuation. It is clearly seen that the inclusion of the impact parameter appreciably changes the inelasticity distribution. Our curve is much flatter compared to the original one. We interpret this as being due to the increase of the small-fireball-formation probability at large impact parameters. The increase of $\chi(k)$ close to $k=1$ is due to the fact that our $c$ in Eq. (8) has been chosen larger than in [2] for the overall fitting. It is manifestly seen that our result is better.

The result of the leading-particle spectrum is compared in Fig. 2 with the experimental data. Again, we see a better agreement of our result with the data as compared with the one obtained in [2]. The effect of small-fireball enhancement is palpable there too.

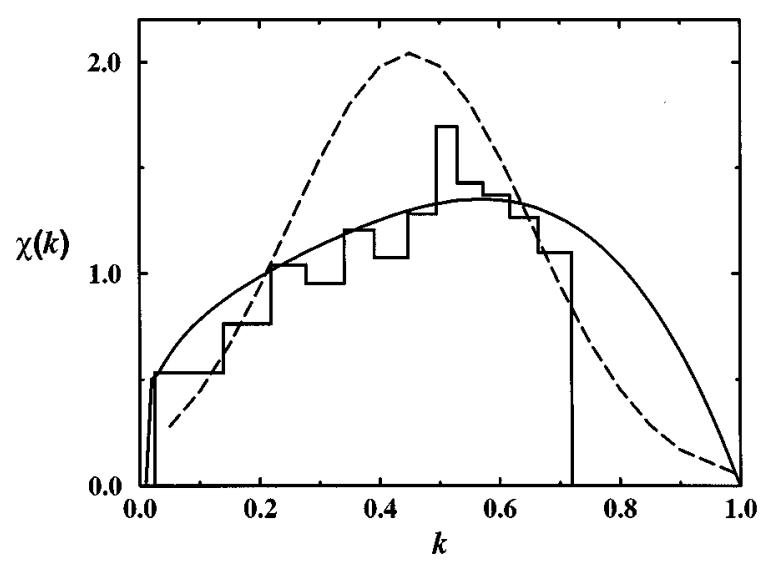

FIG. 1. $\chi(k)$ at $\sqrt{s}=16.5 \mathrm{GeV}$. The data are extracted from [10]. The dashed curve corresponds to the original IGM without impactparameter fluctuation $(c=4)$ and the solid one represents our result $(c=6.4)$.

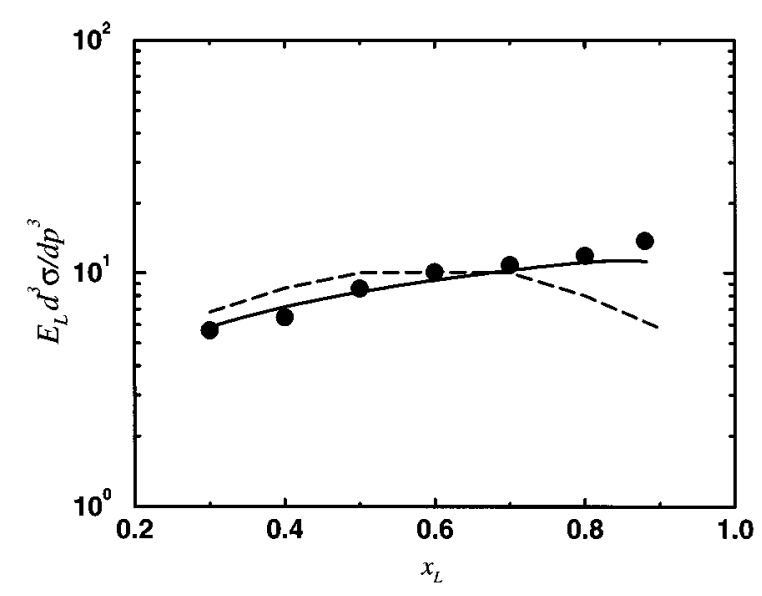

FIG. 2. Leading particle spectrum at $\sqrt{s}=14 \mathrm{GeV}$. The data are extracted from [9] with $p_{T}=0.3 \mathrm{GeV}$. The dashed curve corresponds to the IGM without impact-parameter fluctuation and the solid one represents our result.

\section{PARTICLE SPECTRA}

Having calculated the distribution of fluctuations in the initial conditions, expressed by $\chi(E, P ; \vec{b})$, now we proceed to study their effects on the final particle spectra. For this purpose, we adopt the one-dimensional Landau's hydrodynamical model for an ideal gas. Despite all the simplifications, this model is known to reproduce the main features of the measured momentum (or rapidity) distributions and has advantage of having an analytical solution over the whole rapidity range. The only input of the model is the total energy and the geometrical size of the initial fireball. We will apply this model to the fireballs produced by the gluon fusions as discussed in the previous section. Note that these fireballs do not contain the leading particles anymore. In other words, the hydrodynamics introduced here will not affect the inelasticity calculated in the previous section.

The invariant momentum distribution of produced particles in a hydrodynamical model is usually given by Cooper-Frye formula [11]

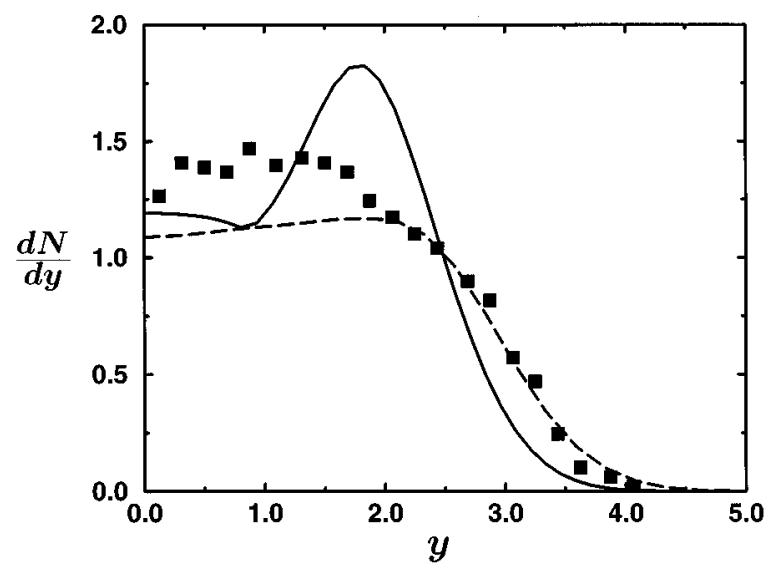

FIG. 3. The rapidity distributions calculated in the usual procedure and with fluctuations at $\sqrt{s}=24 \mathrm{GeV}$. The solid line represents the distribution with the average initial conditions and the dashed line the one obtained with fluctuations. 


$$
E \frac{d N}{d \vec{p}}=\int_{\sigma\left(T_{d}\right)} f\left(p^{\mu} u_{\mu}\right) p^{\mu} d \sigma_{\mu},
$$

where $\sigma\left(T_{d}\right)$ is a constant-temperature freeze-out hypersurface, $p^{\mu}$ is the four momentum of the emitted particle and $u^{\mu}$ is the four velocity of the fluid. Although it is possible to use more realistic freeze-out criteria [12-16], here we limit ourselves to the simplest choice Eq. (33) without sophistication. This will be enough for our present purpose to study how the initial condition fluctuations affect the final particle spectra.

\section{A. Landau's model}

In computing the isothermal $\sigma$ and the velocity (or rapidity) distribution of the fluid in Landau's model, one has to distinguish two different regions. In the so-called nontrivial region $\left[\alpha \leqslant-\xi / \sqrt{3}, \xi=\ln \left(T / T_{0}\right), T_{0}=\right.$ initial temperature, $\alpha=$ fluid rapidity], the temperature $T(x, t)$ and the rapidity $\alpha(x, t)$ are given in terms of the potential [17]

$$
\Psi(\alpha, \xi)=-T_{0} l \sqrt{3} e^{\xi} \int_{\alpha / \sqrt{3}}^{-\xi} e^{2 \xi^{\prime}} I_{0}\left(\sqrt{\xi^{\prime 2}-\alpha^{2} / 3}\right) d \xi^{\prime},
$$

through the relations

$$
\begin{aligned}
& t=\frac{\partial \Psi}{\partial T} \cosh \alpha-\frac{1}{T} \frac{\partial \Psi}{\partial \alpha} \sinh \alpha, \\
& x=\frac{\partial \Psi}{\partial T} \sinh \alpha-\frac{1}{T} \frac{\partial \Psi}{\partial \alpha} \cosh \alpha .
\end{aligned}
$$

Here, we are considering only one hemisphere $\alpha \geqslant 0$. The solution for $\alpha \leqslant 0$ can be obtained by making a reflection. In the simple-wave region,

$$
\alpha=-\frac{\xi}{1 / \sqrt{3}}
$$

and the fluid velocity is related to $(x, t)$ through

$$
x=\frac{v-1 / \sqrt{3}}{1-v / \sqrt{3}} t .
$$

Remember that we are supposing that the fluid is an ideal gas of zero mass particles.

The invariant momentum distribution Eq. (33), with this solution becomes

$$
\begin{aligned}
E \frac{d N}{d \vec{p}}= & \frac{g}{(2 \pi)^{3}} \int_{\sqrt{3} \xi_{d}}^{-\sqrt{3} \xi_{d} m_{T}[\cosh (y-\alpha) \phi(\alpha)+\sinh (y-\alpha) \psi(\alpha)]} \frac{\exp \left\{m_{T} \cosh (y-\alpha) / T_{c}\right\}-1}{g V_{0} e^{-2 \xi_{d}} m_{T}} \\
& \times\left\{\frac{\cosh \left(y-\alpha_{d}\right)+\sqrt{3} \sinh \left(y-\alpha_{d}\right)}{\exp \left\{m_{T} \cosh \left(y-\alpha_{d}\right) / T_{d}\right\}-1}+\frac{\cosh \left(y+\alpha_{d}\right)-\sqrt{3} \sinh \left(y+\alpha_{d}\right)}{\exp \left\{m_{T} \cosh \left(y+\alpha_{d}\right) / T_{d}\right\}-1}\right\},
\end{aligned}
$$

where $V_{0}=l A$ is the initial volume and

$$
\begin{aligned}
& \phi(\alpha)=A \frac{e^{-\xi_{d}}}{T_{0}}\left(\frac{\partial \Psi}{\partial \xi}-\frac{\partial^{2} \Psi}{\partial \alpha^{2}}\right), \\
& \psi(\alpha)=A \frac{e^{-\xi_{d}}}{T_{0}}\left(\frac{\partial \Psi}{\partial \alpha}-\frac{\partial^{2} \Psi}{\partial \alpha \partial \xi}\right)
\end{aligned}
$$

and the suffix " $d$ " stands for dissociation (or freeze-out). In Eq. (39), the first term represents the contribution from the nontrivial region and the second one that from the simple wave.

The formula (39) gives us the invariant momentum distributions of the decay products (pions in the majority) of a fireball in terms of $V_{0}$ and $T_{0}$, once the dissociation temperature $T_{c}$ is fixed. In the original work of Landau, it was assumed that the total energy $\sqrt{s}$ is liberated as thermal energy in a small Lorentz contracted interaction volume

$$
V_{0}=\frac{V}{\gamma},
$$

where $V$ is the proper volume of proton and $\gamma=2 m_{p} / \sqrt{s}$. The initial temperature $T_{0}$ is then computed assuming the fluid is an ideal gas, i.e., $p=\varepsilon / 3$. Nowadays we know that neither the hypothesis of instantaneous thermalization nor the appearance of extremely high values of the initial temperature are physically reasonable so that many people are reluctant to accept the model itself. However, in spite of these rather nonconventional initial conditions, many of the qualitative and the quantitative results (average multiplicity, particle ratios, momentum distributions, ... ) are surprisingly good when compared with data. In our point of view, perhaps the equilibrium is attained at a later time when the system has already suffered some expansion, but then the temperature and the rapidity distributions at the onset of the hydrodynamical regime would be approximately those of Landau's model whose initial conditions correspond to high temperature and energy density if extrapolated back in time. So, for any practical purpose, we can use Landau's solution to describe the system.

Now, as mentioned before, we are going to apply Landau's model to each fireball characterized by its mass $M$ and momentum $\vec{P}$. Then, the total energy is replaced by $M$ and everything is computed in the fireball's rest frame (which is boosted with respect to the center of mass of the collision). But, then which is the initial volume $V_{0}$ in this case? It has been shown [18] that, in the case of the incident-particle fragmentation, 


$$
V_{0}=\frac{V}{\gamma}=\frac{2 m_{p}}{M} V
$$

In the case of the central fireball, we do not have such a simple expression. However, phenomenological analysis [19] of the $M$ dependence of average multiplicity data [20] has shown that in terms of Landau's model those data may well be reproduced if one assumes Eq. (43). Also the $M$ dependence of the momentum distributions, obtained in this way, seem to be consistent with the data [21]. So, in the lack of a better justification founded on a physical basis, we assume Eq. (43) in the present work and compute $T_{0}$ by putting $M$ into this volume. We emphasize, however, that the fluctuation effects which are the central object of the present study do not depend sensibly on such a choice.

\section{B. Rapidity and pseudorapidity distributions}

We now compute the rapidity distributions in two distinct ways. In the first case, with the help of our generator function $\chi(E, P ; \vec{b})$, we calculate the average initial conditions, i.e., the average mass with $\langle P\rangle=0$ because of the symmetry. Then the rapidity distribution is computed as done usually by using the formula (39). In the other case, fluctuations are taken into account and the rapidity distribution given by Eq. (39) is computed for each event and summed over $M, P$ (or $E, P)$ and $\vec{b}$ according to

$$
\begin{aligned}
\frac{\overline{d N}}{d y}= & \int d \vec{b} \int d P \int d E \frac{d N}{d y}(E, P ; \vec{b}) \chi(E, P ; \vec{b}) \\
& \times \theta\left(\sqrt{\left(E^{2}-P^{2}\right) / s}-k_{\min }\right) .
\end{aligned}
$$

The results obtained with these two prescriptions at the incident energy $\sqrt{s}=24 \mathrm{GeV}$ are given in Fig. 3. Observe that, because we have included also the simple wave solution, the rapidity distribution for one fireball with the average initial conditions presents a large peak at this energy. When the fluctuations are taken into account, such a peak is completely smoothed away.

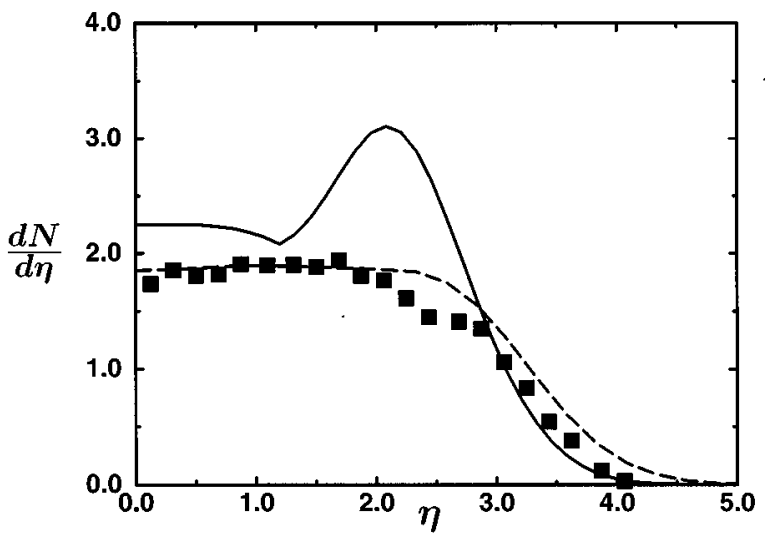

FIG. 4. The pseudorapidity distributions calculated in the usual procedure and with fluctuations at $\sqrt{s}=53 \mathrm{GeV}$. The solid line represents the distribution with the average initial conditions and the dashed line the one obtained with fluctuations. Experimental data [26] are shown for comparison.
Although the main purpose of this work is just to show the influences of the fluctuations in the initial conditions, we may proceed to a comparison with some data [22]. These are given in terms of pseudorapidity. So, we calculate the pseudorapidity distributions for the energies $\sqrt{s}=53$ and 546 $\mathrm{GeV}$, by taking the average value of $m_{T}=0.41$ and 0.49 $\mathrm{GeV}$, respectively. In this approximation the pseudorapidity distribution is given by

$$
\frac{d N}{d \eta}=\frac{d N}{d y} \times \frac{\sqrt{\left(\left\langle p_{T}\right\rangle /\left\langle m_{T}\right\rangle\right)^{2}+\sinh ^{2} y}}{\cosh y} .
$$

The results are shown in Figs. 4 and 5. The comparison of both the results leads us to conclude that the rapidity or equivalently pseudorapidity distributions are very sensitive to the fluctuations in the initial conditions. These fluctuations cause a widening, a smoothing, and a lowering of the distributions. Moreover, if we compare the distributions with and without fluctuations with experimental data, we see that the behavior of the first ones is more similar to the data than the other ones and the presence of the simple-wave peaks in each event does not invalidate the overall agreement with data.

\section{CONCLUDING REMARKS}

We have studied in detail the effects produced by the fluctuations in the initial conditions on the final observables of emitted particles. As a mechanism of fluctuations, we have used a modified version of the interacting gluon model, by including also the impact-parameter fluctuation. The inclusion of the latter has shown to be significant, since a spreading in impact parameter causes a corresponding widening in the fireball mass distribution or, equivalently, in the inelasticity distribution. This widening causes, in its turn, flattening of the leading-particle spectrum. A better agreement of our results with data on these quantities, as compared with the predictions of the original version, may indicate that our version is indeed an improvement.

The modified IGM allowed us to introduce fluctuations in the fireball energy momentum, as well as in the impact pa-

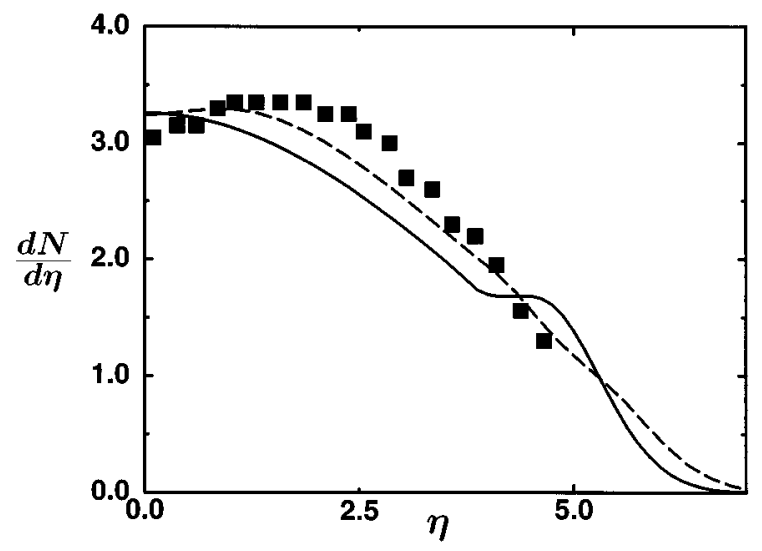

FIG. 5. The pseudorapidity distributions calculated in the usual procedure and with fluctuations at $\sqrt{s}=546 \mathrm{GeV}$. The solid line represents the one with the average initial conditions and the dashed one is the one obtained with fluctuations. Experimental data [22] are shown for comparison. 
rameter, through the distribution function $\chi(E, P ; \vec{b})$. We have then studied the effects of these fluctuations on the rapidity and pseudorapidity distributions, using Landau's hydrodynamical model. This has been done by computing the rapidity (or pseudorapidity) distributions in two distinct ways. First, by the usual procedure in which only one fireball is assumed with the average characteristics (mass, momentum, and impact parameter). Second, by taking the fluctuations into account, by generating each event according to the probability distribution $\chi(E, P ; \vec{b})$ and by summing up over all the events. The difference between them is found to be quite appreciable. As expected, the rapidity (or pseudorapidity) distributions become smoother, wider and lower when fluctuations are considered. The version of hydrodynamical model we used shows peaks in the rapidity distribution (originated from the simple waves) of each event. Nevertheless, in the overall distribution they are entirely smoothed away, showing that even analyses of such a simple quantity as the inclusive one particle distribution may lead to a complete wrong conclusions if fluctuations are totally neglected.

It is well known that there are several observables which cannot be understood if fluctuations are not properly taken into account. For instance, KNO distribution [23], forwardbackward correlation [22], [24], semi-inclusive distributions [22] and so on. However, as is easy to see, the fluctuations we considered in the present work are not enough to account for these quantities. One of the fluctuations we did not include here and which seemingly plays an important role is the multiplicity fluctuation in the fireball decay, given its mass $M$. Investigation in this direction is in progress.

In conclusion, despite all the simplifications made in our description, our results do show that the fluctuations are indeed a very important feature in the hadronic collisions and must be considered in any realistic description of these collisions [25].

\section{ACKNOWLEDGMENTS}

This work has been supported in part by Fundação de Amparo à Pesquisa do Estado de São Paulo (FAPESP) under the contract Nos. 93/2463-2 and 92/1814-3. The authors acknowledge useful discussions with F. S. Navarra concerning the original IGM.
[1] M. Namiki and S. Muroya, "Distribution-Correlation Functions of Produced Particles in High-Energy Nuclear Collisions," Report No. WU-HEP-93-4; M. Namiki and S. Pascazio, Phys. Rep. 232, 301 (1993), see especially subsection 9.4.

[2] G. N. Fowler, F. Navarra, M. Plümer, A. Vourdas, R. M. Weiner, and G. Wilk, Phys. Rev. C 40, 1219 (1989).

[3] For recent applications of the IGM, see for example, F. O. Durães et al., Phys. Rev. D 53, 6136 (1996), and references therein.

[4] L. D. Landau, Izv. Akad. Nauk SSSR 17, 51 (1953).

[5] L. D. Landau and S. Z. Belenkij, Usp. Fiz. Nauk 56, 309 (1956); Nuovo Cimento Suppl. 3, 15 (1956); Collected Papers of L. D. Landau, edited by Ter Haar (Gordon and Breach, New York, 1965).

[6] S. Pokorski and L. van Hove, Acta Phys. Pol. B5, 229 (1974).

[7] T. T. Chou and C. N. Yang, Phys. Rev. 170, 1591 (1968).

[8] R. Castaldi and G. Sanguinetti, Annu. Rev. Nucl. Part. Sci. 35, 351 (1985)

[9] A. M. Rossi et al., Nucl. Phys. B89, 269 (1975).

[10] D. Brick et al., Phys. Lett. 103B, 241 (1981).

[11] F. Cooper and G. Frye, Phys. Rev. D 10, 186 (1974).

[12] K. S. Lee, U. Heinz, and E. Schnedermann, Z. Phys. C 48, 525 (1990).
[13] Y. Hama and F. S. Navarra, Z. Phys. C 53 501, (1992).

[14] F. S. Navarra, M. C. Nemes, S. Paiva, and U. Ornik, Phys. Rev. C 40, 1245 (1993).

[15] F. Grassi, Y. Hama, and T. Kodama, Phys. Lett. B 355, 9 (1995).

[16] F. Grassi, Y. Hama, and T. Kodama, Z. Phys C (to be published).

[17] I. M. Khalatnikov, Zh. Eksp. Teor. Fiz. 27, 529 (1954).

[18] Y. Hama, Phys. Rev. D 19, 2623 (1979).

[19] P. Carruthers and Minh Duong-Van, Phys. Rev. D 28, 130 (1983).

[20] M. Basile et al., Lett. Nuovo Cimento 41, 293 (1984).

[21] M. Basile et al., Phys. Lett. 92B, 367 (1980); Nuovo Cimento 65A, 414 (1981); Nuovo Cimento 67A, 53 (1982); Nuovo Cimento 79A, 1 (1984); Lett. Nuovo Cimento 32, 210 (1981).

[22] UA5 Collaboration, G. J. Alner et al., Phys. Rep. 154, 247 (1987).

[23] Z. Koba, H. B. Nielsen, and P. Olesen, Nucl. Phys. B40, 317 (1972).

[24] S. Uhlig, I. Derado, R. Meinke, and H. Preissner, Nucl. Phys. B132, 15 (1978)

[25] Samya Paiva, Ph.D. thesis, University of São Paulo, 1995.

[26] W. Thomé et al., Nucl. Phys. B129, 365 (1977). 